
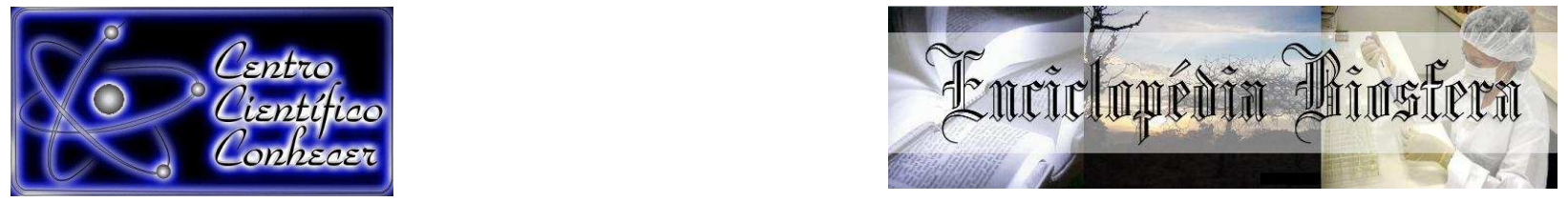

\title{
SÍNDROMES INFECCIOSAS SEXUALMENTE TRANSMISSÍVEIS EM HOMENS: PREVALÊNCIA E FATORES DE RISCO
}

Caio Flávio Castro e Macedo ${ }^{1^{*}}$, Débora Prado Vasconcelos ${ }^{2}$, Patrícia Fernandes de Souza $^{3}$, Thyago Leal Calvo ${ }^{4}$, Geraldo Sadoyama Leal ${ }^{5}$.

*Autor para correspondência

1 Médico Urologista. Mestrado Profissional em Gestão Organizacional, UFG- Regional

Catalão. Av. Dr. Lamartine Pinto de Avelar, 1120 - St. Universitário: Laboratório de Bioquímica e Microbiologia. Catalão - GO, 75704-020, Brasil. (cfcmacedo@gmail.com) Tel. +55 64 99847556

2 Engenheira Biomédica. Mestrado Profissional em Gestão Organizacional, UFG- Regional Catalão. Catalão, Goiás, Brasil.

3 Graduação em Ciências Biológicas. Universidade Federal de Goiás, UFG-Regional Catalão. Catalão, Goiás, Brasil.

4 Graduação em Ciências Biológicas. Universidade Federal de Goiás, UFG-Regional Catalão. Catalão, Goiás, Brasil.

5 Doutor em Imunologia e Parasitologia Aplicadas pela Universidade Federal de Uberlândia. Prof. adjunto III da UFG no curso de Ciências Biológicas-Regional Catalão. Catalão, Goiás, Brasil.

Recebido em: 03/10/2016 - Aprovado em: 21/11/2016 - Publicado em: 05/12/2016 DOI: 10.18677/EnciBio_2016B 192

\section{RESUMO}

Objetivo: estimar a prevalência do diagnóstico sindrômico de DST e analisar os fatores de risco na população masculina atendida em ambulatório público. Métodos: foi realizado um estudo de corte transversal nos anos de 2014 e 2015. A amostra foi constituída por 216 homens entrevistados durante a consulta médica. As análises bivariada e multivariada foram realizadas para a presença de diagnóstico sindrômico e fatores associados. Resultados: aproximadamente um quinto dos homens atendidos apresentou verruga genital. $\mathrm{Na}$ análise bivariada, estado civil e idade constituíram variáveis preditoras para o diagnóstico sindrômico de DST. Já na análise multivariada, apenas o estado civil manteve-se estatisticamente significante como fator de risco para o diagnóstico sindrômico. Conclusões: conclui-se que, apesar de os homens atendidos em um ambulatório especializado referirem ter conhecimento sobre DST, é alto o número de casos dessas patologias. A resistência da população masculina em procurar assistência em saúde reforça a necessidade de estratégias específicas e contínuas para esses indivíduos.

PALAVRAS-CHAVE: diagnóstico sindrômico, doenças sexualmente transmissíveis, fatores de risco.

\section{SEXUALLY TRANSMITTED INFECTIOUS SYNDROMES IN MEN: PREVALENCE AND RISK FACTORS ABSTRACT}

AIMS: To estimate the prevalence of syndromic diagnosis of STD and analyze the risk factors in male population attending a public clinic. METHODS: We conducted a cross-sectional study in the years 2014 and 2015. The sample consisted of 216 men 
interviewed during the medical consultation. The bivariate and multivariate analyzes were performed for the presence of syndromic diagnosis and associated factors. RESULTS: Approximately one-fifth of treated men had genital wart. In the bivariate analysis, marital status and age were predictors for syndromic diagnosis of STD. In the multivariate analysis, only marital status remained statistically significant as a risk factor for the syndromic diagnosis. CONCLUSIONS: We conclude that, although men treated at a specialized clinic refer having knowledge about STD, is high number of cases of these diseases. The strength of the male population to seek health care reinforces the need for specific and ongoing strategies for these individuals.

KEYWORDS: syndromic diagnosis, sexually transmitted diseases, risk factors.

\section{INTRODUÇÃO}

As doenças sexualmente transmissíveis (DSTs) são consideradas um dos problemas de saúde pública mais comuns em todos os continentes, além de tornarem o organismo mais suscetível à infecção por HIV. Não obstante, estão relacionadas com a mortalidade materno-infantil (WAGNER et al., 2013).

Em todo o mundo, estima-se o diagnóstico de 448 milhões de novos casos de DST curáveis por ano, com sequelas evitáveis quando a abordagem é feita no estágio inicial (WAGNER et al., 2013). Aproximadamente 34,2 milhões de pessoas estão infectadas por HIV em todos os continentes, com 2,5 milhões de novos casos a cada ano (TODD et al., 2012), sendo que a Organização Mundial de Saúde (OMS) estima aproximadamente 5.772 .000 casos anuais de DST no Brasil (BRASIL, 2015).

O diagnóstico etiológico das DSTs antes de se iniciar o tratamento nem sempre é possível, e o manejo sindrômico da doença envolve a identificação de um conjunto de sinais e sintomas, além do tratamento contra o organismo mais comum (SEN et al., 2013). Enquanto o diagnóstico laboratorial e etiológico é considerado o padrão ouro para o manejo das infecções sexualmente transmissíveis, a abordagem sindrômica tem sido apresentada como uma alternativa simplificada e acessível para ambientes de recursos limitados (MEDRONHO et al., 2009; KHAN et al., 2014).

O diagnóstico sindrômico é recomendado pelo Ministério da Saúde brasileiro e também em outros países pobres ou em desenvolvimento, como a Índia (PRABHAKAR et al., 2012). Para o diagnóstico etiológico, o SUS disponibiliza ainda as sorologias para sífilis, HIV, hepatites $B$ e $C$, considerando a prevalência e morbidade (PAN et al., 2013).

As DSTs de notificação compulsória são as Hepatites Virais, a infecção pelo HIV em gestantes e crianças expostas ao risco de transmissão vertical, a Sífilis e a AIDS (BRASIL, 2012 a). A ausência de notificação regular das demais doenças dificulta o reconhecimento do real estado entre a população, além de prejudicar as ações institucionais para prevenção e controle (BRASIL, 2012 b; BRASIL, 2015). Portanto, identificar as particularidades dos homens portadores de DST é fundamental para subsidiar estratégias públicas de prevenção para tais enfermidades. Assim, o objetivo deste estudo foi caracterizar os homens portadores de DSTs atendidos em ambulatório público especializado e os fatores de risco associados.

\section{MATERIAL E MÉTODOS}

Tratou-se de uma pesquisa de corte transversal, do tipo não controlada, acerca da exposição aos fatores de risco. A unidade de análise foi o indivíduo, com esquema de seleção completo e amostra de conveniência (MEDRONHO et al., 2009). 
A população do trabalho constituiu-se de 216 homens encaminhados ou que procuraram espontaneamente o ambulatório de DST masculino. A amostra incluiu indivíduos acima de 14 anos que autorizaram a coleta de dados, ou que tiveram autorização do responsável, no caso de menores de 18 anos, independentemente da orientação sexual.

As informações foram coletadas entre junho de 2014 e fevereiro de 2015 na cidade de Catalão, GO. O instrumento de coleta de dados (ficha clínica) foi aplicado pelo investigador, médico urologista, e respondido pelo entrevistado após sua devida orientação. Houve paridade entre o sexo do pesquisador e o dos participantes, o que pode minimizar a resistência masculina na abordagem de questões de saúde, em especial no que se refere ao comportamento sexual.

$\mathrm{Na}$ ficha clínica constavam as seguintes variáveis preditoras: idade, estado civil, escolaridade, renda familiar, idade de início das atividades sexuais, prática sexual, comportamento sexual, número de parceira(os), parceira(o) fixa, uso de preservativo, pagamento para manter contato sexual, conhecimento sobre transmissão de DST, uso de drogas ilícitas, postectomia e diagnóstico prévio de DST. Essas variáveis foram testadas em relação ao desfecho de diagnóstico sindrômico e foram elaboradas a partir dos possíveis fatores de risco e proteção relacionados ao tema (GUO et al., 2011; FAN et al., 2012; PAN et al., 2013; WAGNER et al., 2013).

As variáveis sociodemográficas idade, classe social e escolaridade foram então definidas. Para isso, de acordo com o Estatuto da Juventude brasileiro, são consideradas jovens as pessoas entre 15 e 29 anos de idade (BRASIL, 2013). Enquanto que a divisão de classes sociais por renda familiar e a de escolaridade foram determinadas com base no Instituto Brasileiro de Geografia e Estatística e na Lei de Diretrizes e Bases da Educação Nacional, respectivamente (IBGE, 2010; BRASIL, 1996). Um estudo apontou uma média de idade da primeira relação do brasileiro como 15,7 anos (HUGO et al., 2011), o que permitiu definir essa variável comportamental com o corte em 16 anos.

O diagnóstico sindrômico foi determinado pela história clínica e exame físico genital do paciente, com a presença de úlcera, verruga genital e secreção em meato uretral (BRASIL, 2006; PRABHAKAR et al., 2012). O pesquisador e um auxiliar de investigação realizaram a dupla digitação dos dados e a conferência acerca da consistência do banco. O conteúdo foi analisado no programa estatístico SPSS (Statistical Package for Social Sciences for Windows) versão 20.0 (MAROCO, 2011).

Realizou-se uma análise estatística descritiva para determinar as frequências relativas e absolutas, e o teste do qui-quadrado $\left(\square^{2}\right)$ para comparação entre os valores percentuais (variáveis qualitativas). A força de associação entre cada uma das variáveis explicativas e a variável resposta foi avaliada pelo cálculo de razão de prevalência (RP), acompanhado do respectivo intervalo de confiança de 95\% (IC 95\%). Em todas as análises foram consideradas como significantes os resultados que apresentaram um nível de significância (a) de 5\%, ou seja, p-valor igual ou inferior a $5 \%(\leq 0,05)$.

A análise multivariada entre a presença de diagnóstico sindrômico e possíveis fatores associados foi realizada por meio de modelos lineares generalizados, usando-se a distribuição de Poisson com função de ligação logarítmica e variância robusta para aproximar à binomial. Para a construção desse modelo foram incluídas as variáveis que obtiveram valor de $p$ menor ou igual a 0,20 na análise bivariada.

O projeto foi aprovado pelo Comitê de Ética em Pesquisa (CEP) sob o 
parecer número 640.871, e a concessão de informações aos participantes acerca do trabalho de investigação constou no Termo de Consentimento Livre e Esclarecido e Termo de Assentimento para menores de 18 anos, os quais foram devidamente assinados.

Aos homens que foram diagnosticados com qualquer das DSTs pesquisadas durante o trabalho foram ofertados tratamento e acompanhamento por equipe de médico e enfermeiro da Secretaria Municipal de Saúde de Catalão, através do Sistema Único de Saúde (SUS).

\section{RESULTADOS}

Dos 216 homens atendidos em um ambulatório público de urologia, 7,9\% $(n=17)$ foram diagnosticados com síndrome uretral, 13,9\% $(n=30)$ com síndrome ulcerosa e 20,4\% ( $n=44)$ com síndrome verrucosa. Pouco mais da metade da população estudada tem mais de 29 anos de idade e $40,48 \%$ são casados. Em relação à escolaridade, $37,5 \%$ dos homens cursaram até o ensino fundamental, $43,5 \%$ concluíram o ensino médio, e apenas dois pacientes declarados como analfabetos. Portanto, de acordo com a classificação do IBGE, dois terços dos homens atendidos encontram-se na Classe D.

Aproximadamente metade dos pacientes relataram ter tido a primeira relação sexual com menos de 16 anos de idade. Já em relação à prática sexual, a quase totalidade relatou sexo vaginal, metade oral e um terço anal. Além disso, $90,7 \%$ referiram comportamento heterossexual, 5,6\% bissexual e apenas cinco (5) homens relataram comportamento homossexual.

Quanto ao número de parceiras, quase dois terços tiveram duas ou mais parceiras nos últimos 12 meses e 65,7\% relataram ter parceira fixa. Considerou-se como uso de preservativo quando esse foi utilizado durante todo o ato sexual e em todas as relações, nos últimos 12 meses. Apenas 12\% dos homens responderam "sim" de acordo com esse critério. Pouco mais de dois terços dos indivíduos mencionaram ter algum conhecimento sobre DST e $82,2 \%$ negaram diagnóstico prévio de tais doenças.

Dos participantes, 12\% aludiram uso de alguma droga ilícita e o mesmo número de entrevistados relatou ter efetuado pagamento para manter relação sexual no último ano. Apenas $7,9 \%$ dos homens foram submetidos à postectomia. Dados esses amostrados na Tabela 1.

TABELA 1. Caracterização geral da população de Catalão ( $n=216)$, GO, 2015.

\begin{tabular}{lcc}
\hline Características & $\mathrm{N}$ & $\%$ \\
Idade $\leq 29$ anos & 102 & 47,4 \\
Idade > 29 anos & 113 & 52,9 \\
Casado & 85 & 40,48 \\
Não casado & 125 & 59,52 \\
Escolaridade & & \\
Analfabeto & 2 & 0,9 \\
Ensino fundamental & 118 & 54,9 \\
Ensino médio & 83 & 38,6 \\
Ensino superior & 11 & 5,1 \\
Pós-graduação & 1 & 0,5
\end{tabular}




\begin{tabular}{|c|c|c|}
\hline $\begin{array}{l}\text { Renda Familiar } \\
\text { Classe A: Acima de } R \$ 15.300,00 \\
\text { Classe B: de } R \$ 7.650,00 \text { até } R \$ 15.300,00 \\
\text { Classe C: de } R \$ 3.060,00 \text { até } R \$ 7.650,00 \\
\text { Classe D: de } R \$ 1.020,00 \text { até } R \$ 3.060,00 \\
\text { Classe E: Até } R \$ 1.020,00\end{array}$ & $\begin{array}{c}1 \\
17 \\
28 \\
144 \\
20\end{array}$ & $\begin{array}{c}0,48 \\
8,1 \\
13,29 \\
68,6 \\
9,53\end{array}$ \\
\hline $\begin{array}{l}\text { Idade da primeira relação sexual } \\
<16 \text { anos } \\
\geq 16 \text { anos }\end{array}$ & $\begin{array}{l}112 \\
102\end{array}$ & $\begin{array}{l}52,34 \\
47,66\end{array}$ \\
\hline $\begin{array}{l}\text { Prática Sexual } \\
\text { Vaginal } \\
\text { Oral } \\
\text { Anal }\end{array}$ & $\begin{array}{l}206 \\
103 \\
56\end{array}$ & $\begin{array}{l}96,3 \\
48,1 \\
26,2\end{array}$ \\
\hline $\begin{array}{l}\text { Comportamento Sexual } \\
\text { Heterossexual } \\
\text { Homossexual } \\
\text { Bissexual }\end{array}$ & $\begin{array}{l}195 \\
5 \\
12\end{array}$ & $\begin{array}{l}92 \\
2,4 \\
5,6\end{array}$ \\
\hline $\begin{array}{l}\text { Número de Parceiras } \\
\text { Nenhuma } \\
\text { Única } \\
\text { Múltipla }\end{array}$ & $\begin{array}{l}6 \\
65 \\
129\end{array}$ & $\begin{array}{c}3 \\
32,5 \\
64,5\end{array}$ \\
\hline $\begin{array}{l}\text { Parceira fixa } \\
\text { Uso de Preservativo }\end{array}$ & $\begin{array}{l}141 \\
26\end{array}$ & $\begin{array}{l}67,8 \\
12,3\end{array}$ \\
\hline $\begin{array}{l}\text { Pagamento para manter contato sexual } \\
\text { Conhecimento Sobre Transmissão de DST } \\
\text { Fez uso de Alguma Droga llícita nos Últimos } \\
12 \text { Meses }\end{array}$ & $\begin{array}{l}31 \\
161 \\
26\end{array}$ & $\begin{array}{l}14,4 \\
74,5 \\
12,2\end{array}$ \\
\hline $\begin{array}{l}\text { Postectomia } \\
\text { Diagnóstico Prévio de DST }\end{array}$ & $\begin{array}{l}17 \\
45\end{array}$ & $\begin{array}{l}7,9 \\
21\end{array}$ \\
\hline
\end{tabular}

FONTE: Os autores, (2015).

$\mathrm{Na}$ análise bivariada, estado civil e idade constituíram variáveis preditoras para o diagnóstico sindrômico de DST (Tabela 2). Foram consideradas para a análise multivariada as variáveis independentes que apresentarem $n$ ível de significância $(p)$ $\leq 20 \%$ : idade, estado civil, idade da primeira relação sexual, renda familiar, número de parceiras, conhecimento sobre transmissão de DST e uso de drogas ilícitas nos últimos 12 meses. Após essa análise, apenas o estado civil manteve-se estatisticamente significante como fator de risco para alguma síndrome infecciosa (Tabela 3).

TABELA 2. Síndrome x Não Síndrome (análise bivariada). Catalão, GO, 2015. Características Síndrome

Sim N Não N p; RP (IC)

Idade

(\%) (\%)

Idade $>29$

$34 \quad 79$

1 


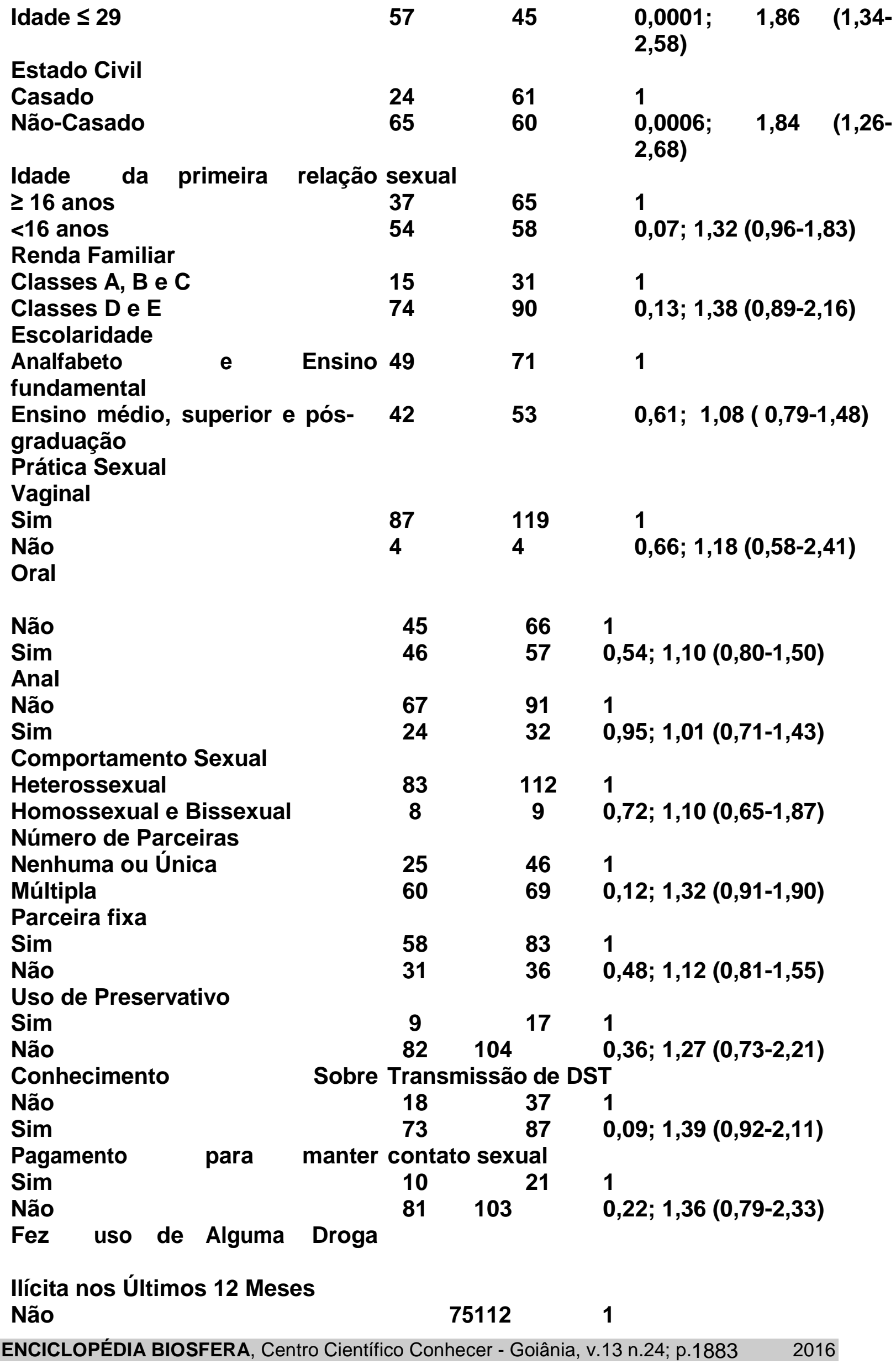


Sim

Postectomia

Não

Sim

Diagnóstico Prévio de DST

Sim

Não

FONTE: Os autores, (2015).
$15 \quad 11 \quad 0,08 ; 1,43(0,99-2,08)$

$82115 \quad 1$

$9 \quad 8 \quad 0,36 ; 1,27(0,78-2,05)$

$18 \quad 27 \quad 1$

$73 \quad 96 \quad 0,70 ; 1,08(0,72-1,60)$

TABELA 3. Síndrome x Não Síndrome (análise multivariada). Catalão, GO, 2015.

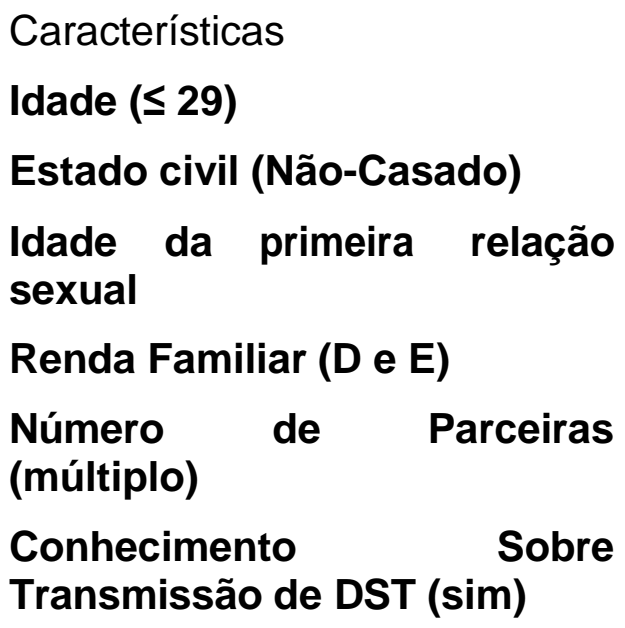

$\mathrm{p} \quad \mathrm{RP}$

0,087

0,024

1,098

1,153

1,093

1,079

0,934

0,224

0,285

0

0,191

1,083

Fez uso de Alguma Droga Ilícita nos Últimos 12 Meses (sim)

0,512
$0,961-1,219$

IC (95\%)

$0,987-1,221$

$1,019-1,305$

$0,990-1,206$

$0,955-1,218$

$0,823-1,059$

$0,911-1,206$

FONTE: Os autores, (2015).

\section{DISCUSSÃO}

A alta prevalência do diagnóstico sindrômico no presente trabalho, em especial de verruga genital, é explicada por tratar-se de uma população atendida em um ambulatório criado para acolher homens com queixas, dúvidas ou mesmo suspeitas de DST. Mesmo considerando essa limitação, esse achado é corroborado por outros trabalhos científicos (MAKASA et al., 2012; CAVALCANTI et al., 2012; GOMES et al., 2013).

A maioria dos homens entrevistados tinham mais de 29 anos, completou o ensino médio ou fundamental, pertencem às classes sociais $C$ ou $D$, relataram ter comportamento heterossexual e conhecimento prévio sobre transmissão de DST. Outras pesquisas demonstraram diferenças em relação às características citadas acima, nas populações estudadas (GUO et al., 2011; MAKASA et al., 2012). Por outro lado, neste estudo, a maior parte dos indivíduos atendidos eram solteiros, mantiveram a primeira relação sexual com menos de 16 anos, têm múltiplas parceiras e não usam preservativos, particularidades comuns aos estudos epidemiológicos de doenças sexualmente transmissíveis (FAN et al., 2012).

Apesar da maioria dos homens entrevistados relatarem ter conhecimento sobre o tema abordado, apenas uma minoria assumiu utilizar o preservativo em toda relação e durante todo o ato sexual. Ainda, a maioria dos indivíduos responderam ter uma parceira fixa, mas também ter mais de uma parceira. Esses 
dados apoiam a ideia de que mesmo referindo ter informação sobre as DSTs, a população masculina tem uma baixa aderência às medidas de prevenção, o que aumenta a chance de adquirir tais doenças.

Nos países em desenvolvimento, estudo apontou que constituem fatores de risco para DST o sexo masculino e a população jovem, em especial com menos de 15 anos. Além disso, a crescente urbanização, o analfabetismo, pobreza, promiscuidade sexual, a falta de educação em saúde e a resistência aos fármacos disponíveis para tratamento são apontados como justificativas para a alta prevalência dessas patologias (SEN, 2013).

Em todo o mundo, e também no Brasil, as epidemias de DST e HIV são altamente concentradas entre homens que fazem sexo com homens (HSH) (MALTA et al., 2015), indicando o comportamento homo ou bissexual como fator de risco. $E$ define-se comportamento sexual de alto risco a atividade sexual com um parceiro casual ou comercial sem o uso de um preservativo de forma consistente (TODD et al., 2012). Dos homens avaliados na presente pesquisa, a quase totalidade respondeu ter comportamento heterossexual, diferença que pode ser explicada em parte pelo viés de algum constrangimento na resposta dessa questão.

Os fatores sociodemográficos: idade, escolaridade e situação financeira são apontados como fatores de risco para a infecção pelo HIV ou outras doenças sexualmente transmissíveis. Ainda como variáveis preditoras para DST estão: a duração do trabalho sexual, padrões de migração, consumo de álcool e outras drogas, relação sexual anal passiva, história de DST prévia (GUO et al., 2011; PAN et al., 2013), presença de úlcera genital, nunca ter feito sexo com uma mulher, não conhecer o status de HIV do parceiro masculino mais recente e nunca ter comprado preservativos nos últimos 12 meses (FAN et al., 2012).

Em homens norte-americanos que pagam para manter relações sexuais com mulheres, os testes positivos para HIV e demais DSTs foram significativamente associados ao comportamento bissexual, uso de drogas injetáveis e cocaína, nos últimos quatro meses (WAGNER et al., 2013).

$\mathrm{Na}$ Holanda, uma pesquisa avaliou 659 HSH e encontrou uma prevalência de $16,0 \%$ de DSTs assintomáticas, principalmente sífilis e clamídia na região anal. Por análise multivariada, qualquer DST foi associada com idade do paciente abaixo de 40 anos, ter tido relações sexuais com dois ou mais parceiros, uso de brinquedos sexuais com um companheiro e uso de enema antes da relação sexual (HEILIGENBERG et al., 2012).

Em uma clínica de doenças sexualmente transmissíveis de Manaus, Brasil, foram avaliadas 434 amostras de úlceras genitais, $84,8 \%$ de homens. O DNA do Herpes Simplex Virus (HSV) tipo 2 foi detectado em 55,3\% das amostras, Treponema pallidum em 8,3\%, HSV tipo 1 em 3,2\%, e nenhum caso de Haemophilus ducreyi foi identificado. As sorologias para HIV e sífilis foram positivas em $3,2 \%$ e $5,8 \%$ dos pacientes, respectivamente (GOMES et al., 2013). Em um estudo semelhante realizado no continente africano, apenas $45 \%$ dos pacientes tiveram o diagnóstico etiológico estabelecido (MAKASA et al., 2012), o qual demonstra limitações da abordagem sindrômica.

Outro estudo brasileiro avaliou a prevalência de síndrome infecciosa em homens e mulheres atendidos em um centro primário de saúde. A síndrome mais frequente foi verruga genital $(42,2 \%)$. Úlceras e verrugas genitais foram mais prevalentes em homens, que também relataram ter mais parceiros sexuais do que as mulheres (CAVALCANTI et al., 2012). No presente manuscrito, a síndrome com maior prevalência foi também a verrucosa, o que justifica a implementação de 
esforços para um acompanhamento adequado desses casos.

O Ministério da Saúde realizou um estudo multicêntrico em seis capitais brasileiras e identificou prevalência de DST curáveis (sífilis, gonorreia e clamídia) em $6,2 \%$ dos trabalhadores da indústria, e $19,7 \%$ das pessoas que frequentam clínicas especializadas em DST (BRASIL, 2006). Uma prevalência superior a $5 \%$ das DST curáveis determina uma enorme carga de morbidade e fortalece as evidências de falhas nas estratégias de prevenção e controle no Brasil (BRASIL, 2006; CAMPOS et al., 2010).

Para populações de risco, apenas os sintomas auto relatados não podem ser considerados uma estratégia de rastreio eficaz e os programas de prevenção de DST devem se concentrar na identificação dos sintomas, como também considerar exames de rotina e de referência para esse grupo (WAGNER et al., 2013). Não obstante, os pacientes assintomáticos são desconsiderados na abordagem sindrômica, tornando-a insuficiente para combater sozinha a ameaça constante da propagação de DST e HIV (SEN, 2013).

$\mathrm{Na}$ análise bivariada, a idade constituiu variável preditora para o diagnóstico sindrômico de DST e está de acordo com outros trabalhos publicados (GUO et al., 2011; FAN et al., 2012; PAN et al., 2013; WAGNER et al., 2013). Por outro lado, apenas o estado civil manteve-se estatisticamente significante como fator de risco nas análises bivariada e multivariada. Essa é uma observação diferente em comparação aos dados literários encontrados, que apontaram idade, escolaridade e situação financeira como fatores sociodemográficos de risco para alguma DST (GUO et al., 2011; SEN, 2013). Essa disparidade pode ser parcialmente explicada por tratar-se de amostra exclusiva de homens atendidos em ambulatório especializado, além do desfecho pesquisado ser definido como síndrome infecciosa e não como diagnóstico etiológico (MAKASA et al., 2012; CAVALCANTI et al., 2012; GOMES et al., 2013).

Aponta-se como limitações da pesquisa: o desenho transversal, a amostra de conveniência e a subjetividade de entrevistas como ferramenta de estudo. Ainda assim, os dados encontrados permitem oferecer subsídios para ações preventivas de educação popular em saúde.

\section{CONCLUSÃO}

Conclui-se que é alto o número de casos de DST em homens atendidos em um ambulatório especializado, sendo que o conhecimento sobre o tema não repercutiu em medidas de cuidado como o uso do preservativo. Ainda, associado aos dados obtidos, a resistência masculina em procurar assistência em saúde reforça a necessidade de estratégias específicas e contínuas para prevenção, rastreamento, diagnóstico e tratamento precoce nesse grupo populacional.

\section{AGRADECIMENTOS}

À Fundação de Amparo à Pesquisa do Estado de Goiás (FAPEG) e Universidade Federal de Goiás (UFG) - Regional Catalão.

\section{CONFLITO DE INTERESSES}

Os autores deste manuscrito declaram a inexistência de conflito de interesses em relação ao presente estudo. 


\section{SUPORTE FINANCEIRO}

Primeiro autor é bolsista pela Fundação de Amparo à Pesquisa do Estado de Goiás (FAPEG).

\section{REFERÊNCIAS}

BRASIL, Lei no 9.394, de 20 de dezembro de 1996 [internet]. Brasília: Presidência da República Casa Civil; 1996 dec 20 [citado 2015 sep 20].

Disponível em: <http://www.planalto.gov.br/ccivil_03/LEIS/L9394.htm>.

BRASIL, Lei no 12.852, de 5 de agosto de 2013 [internet]. Brasília: Presidência da República; 2013 Aug 5 [citado 2015 sep 20]. Disponível em: http://www.planalto.gov.br/ccivil_03/_Ato2011-2014/2013/Lei/L1282.htm.

BRASIL, Boletim epidemiológico hepatites virais ano III no. 1 [internet]. Brasília: Ministério da Saúde; 2012 a [citado 2015 sep 20]. Disponível em: http://www.aids.gov.br/sites/def

ault/files/anexos/publicacao/2012/51820/boletim_epidemiol_gico_hepatites_virais_20 12_ve_12026.pdf

BRASIL, Boletim epidemiológico sífilis ano I no. 01 [internet]. Brasília:Ministério da Saúde; 2012 b [citado 2015 sep 20]. Disponível em: http://www.dst.uff.br/publicacoes/Boletim_epidem_sifilis_2012.pdf

BRASIL, Programa Nacional de DST e Aids. Manual de controle das doenças sexualmente transmissíveis [internet]. 4th ed. Brasília: Ministério da Saúde; 2006 [citado em 2015 sep 20].

Disponível em: http://bvsms.saude.gov.br/bvs/publicacoes/manual_controle_das_dst. pdf.

CAMPOS, A.L.A.; ARAÚJO, M.A.L.; MELO, S.P.; GONÇALVES M.L.C. Epidemiology of gestational syphilis in Fortaleza, Ceará State, Brazil: an uncontrolled disease. Caderno de Saúde Pública, v, 26, n. 9, p. 1747-1755, 2010. Disponível em: http://dx.doi.org/10.1590/S0102-311X2010000900008

CAVALCANTI, E.G.F.; ARAÚJO, M.A.L.; GALVÃO, M.T.G.; MOURA, H.J.; GONDIM, A.P.S.; SILVA, R.M. Sexually transmitted infections associated syndromes assisted in the primary health care in Northeast, Brazil. BMC Public Health, v. 12, 2012. DOI:10.1186/1471- 2458-12-595.

FAN, S., LU, H.; MA, X.; SUN, Y.; HE, X.; LI, C.; RAYMOND, H.F.; McFARLAND, W.; SUN, J.; MA, W.; JIA, Y.; XIAO, Y.; SHAO, Y.; RUAN, Y. Behavioral and serologic survey of men who have sex with men in Beijing, China: implication for HIV intervention. AIDS Patient Care STDS, v. 26, n. 3, 2012. DOI: 10.1089/apc.2011.0277.

GOMES, N.F.; SABIDÓ, M.; AMARAL, P.A.T.; ARAÚJO, V.E.; CONTRERAS, M.M.C.; GALBAN, E.; BENZAKEN, A.S. Etiology of genital ulcer disease in a sexually transmitted infection reference center in Manaus, Brazilian Amazon. PLoS One, v. 21, n. 5, 2013. DOI:10.1371/journal.pone.0063953. 
GUO, Y.; LI, X.; FANG, X.; LIN, X.; SONG, Y.; JIANG, S.; STANTON, B. A comparison of four sampling methods among men having sex with men in China: implications for HIV/STD surveillance and prevention. AIDS Care, v. 23, n. 11, p. 1400-9, 2011. DOI: 10.1080/09540121.2011.565029.

HEILIGENBERG, M.; RIJNDERS, B.; SCHIM van der LOEFF, M.F.; de VRIES, H.J.; van der MEIJDEN, M.I.; GEERLINGS, S.E.; FENNEMA, H.S.; PRINS, M.; PRINS J.M. High prevalence of sexually transmitted infections in HIV- infected men during routine outpatient visits in the Netherlands. Sexually Transmitted Diseases, v. 39, n. 1, p. 815, 2012. DOI: 10.1097/OLQ.0b013e3182354e81.

HUGO, T.D.O.; VANESSA, T. M.; KAREN, J.; RODRIGUES, C. E. G.; CRUZEIRO, A. L. S.; ORES, L. C.; PINHEIRO, R. T.; SILVA, R.; SOUZA, L. D. M. Fatores associados à idade da primeira relação sexual em jovens: estudo de base populacional [Associated factors to age of first sexual intercourse among young: a population base study]. Caderno de Saúde Pública, v. 27, n. 11, p. 2207-2214, 2011. Disponível em: http://dx.doi.org/10.1590/S0102-311X2011001100014.

IBGE - Indicadores Sociais Municipais: uma análise dos resultados do universo do Censo Demográfico [internet]. 2010 [citado 2015 Sep 19]. Disponível

em: http://www.ibge.gov.br/home/estatistica/populacao/censo2010/indicadores_socia is_municipais/indicadores_sociais_municipais_tab_pdf.shtm.

KHAN, M.A. JAVED, W.; AHMED, M.; WALLEY, J.; MUNIR, M.A. Sexually transmitted disease syndromic case management through public sector facilities: development and assessment study in punjabpakistan. Ann Glob Health, v. 80, n. 6, 2014. DOI: 10.1016/j.aogh.2015.02.002.

MAKASA, M; BUVE, A; SANDOY, I.F. Etiologic pattern of genital ulcers in Lusaka, Zambia: has chancroid been eliminated. Sexually Transmitted Diseases, v. 39, n. 10, 2012. DOI: 10.1097/OLQ.0b013e31826ae97d

MALTA, M.; SILVA, C.M.F.P.; MAGNANINI, M.M.F.; WIRTZ, A.L.; PERISSÉ, A.R.S.; BEYRER, C.; STRATHDEE, S.A.; BASTOS, F.I. Improvement of HAART in Brazil, 1998-2008: a nationwide assessment of survival times after AIDS diagnosis among men who have sex with men. BMC Public Health, v. 15, n. 1, 2015. DOl: 10.1186/s12889-015-1530-y.

MAROCO, J. Análise estatística com SPSS Statistics. 5th ed. Pero Pinheiro: Report Number; 2011.

MEDRONHO, R.A; BLOCK, K.V; LUIZ, R.R; WERNWCK, G.L. Epidemiologia. 2nd ed. Rio de janeiro: Atheneu; 2009.

PAN, X.; ZHU, Y.; WANG, Q.; ZHENG, H.; CHEN, X.; SU, J.; PENG, Z.; YU, R.; WANG N. Prevalence of HIV, syphilis, HCV and their high risk behaviors among migrant workers in eastern China. PLoS One, v. 8, n; 2, 2013. DOI: 10.1371/journal.pone.0057258. 
PRABHAKAR, P.; NARAYANAN, P.; DESHPANDE, G.R.; DAS, A.; NEILSEN, G.; MEHENDALE, S.; RISBUD, A. Genital ulcer disease in India: etiologies and performance of current syndrome guidelines. Sexually Transmitted Diseases, v. 39, n. 11, 2012. DOI: $10.1097 / \mathrm{OLQ} .0 \mathrm{~b} 013 \mathrm{e} 3182663 \mathrm{e} 22$.

SEN, S. Syndromic management in the control of sexually transmitted infections: time for a relook. Indian J Dermatol Venereol Leprol, v. 79, n. 6, 2013. DOI: 10.4103/0378-6323.120738.

TODD, C.S; NASIR, A.; MANSOOR, G.F.; SAHIBZADA, S.M.; JAGODZINSKI, L.L.; SALIMI, F.; KHATERI, M.N.; HALE, B.R.; BARTHEL, R.V.; SCOTT, P.T. Crosssectional assessment of prevalence and correlates of blood-borne and sexually transmitted infections among Afghan National Army recruits. BMC Infect Dis, v. 12, 2012. DOI: 10.1186/1471-2334-12-196.

WAGNER, K.D.; PITPITAN, E.V.; CHAVARIN, C.V.; MAGIS-RODRIGUEZ, C.; PATTERSON, T.L. Drug- using male clients of female sex workers who report being paid for sex: HIV/sexually transmitted infection, demographic, and drug use correlates. Sexually Transmitted Diseases, v. 40, n. 8, 2013. DOI: 10.1097/OLQ.0b013e31829569ec. 